\title{
Functional Electrical Stimulation (FES) to the Biceps Brachii for Controlling Forearm Supination in the Paralyzed Upper Extremity
}

\author{
Akira Naito, Michihiro Yajima*, Hidehiko \\ Fukamachi*, Koji Ushikoshi†, Yasunobu Handa $\ddagger$, \\ Nozomu Hoshimiya and Yoshifusa Shimizu \\ Department of Anatomy, Shinshu University School of \\ Medicine, Matsumoto 390, *Kosei-Ren Kakeyu-Misayama \\ Hospital, Marko-Machi 386-03, † Aizawa Hospital, \\ Matsumoto 390, $¥$ Department of Anatomy, Tohoku \\ University School of Medicine, Sendai 980-77, and \\ §Department of Electronic Engneering, Faculty of \\ Engneering, Tohoku University, Sendai 980-77
}

Naito, A., Yajima, M, Fukamachi, H., Ushikoshi, K., Handa, Y., Hoshimiya, N. and Shimizu, Y. Functional Electrical Stimulation (FES) to the Biceps Brachii for Controlling Forearm Supination in the Paralyzed Upper Extremity. Tohoku J. Exp. Med., 1994, 173 (2), 269-273_ Control of forearm supination using the functional electrical stimulation (FES) to the biceps brachii was studied in a C4 quadriplegic patient. As a result of FES to the biceps brachii, and the other elbow flexors and extensors, supination was induced in maintenance of the elbow in the maximum flexion and extension, respectively. Although a range of the movements was still small and limited, these findings indicated a possibility of FES to the biceps brachii for controlling supination in paralyzed upper extremities. - functional electrical stimulation (FES); biceps brachii; elbow flexors and extensors; forearm supination; paralyzed upper extremity

In order to restore motor functions of paralyzed extremities using the functional electrical stimulation (FES), we have developed a computer controlled multi-channel FES system and percutaneous intramuscular electrodes for selective activations of paralyzed muscles (Handa and Hoshimiya 1987; Handa et al. 1989; Hoshimiya et al. 1989). When we controlled forearm supination in paralyzed upper extremities with these apparatuses, we tried to activate the supinator. However, FES to the supinator always induced simultaneous activations of the posterior interosseus nerve, which tunnels through the muscle, and resulted in extension of the wrist, finger and thumb, and abduction of the thumb in addition to supination of the forearm (Fig. 1).

The biceps brachii, which belongs to the elbow flexors, is known as a strong

Received February 21, 1994; revision accepted for publication May 5, 1994. 

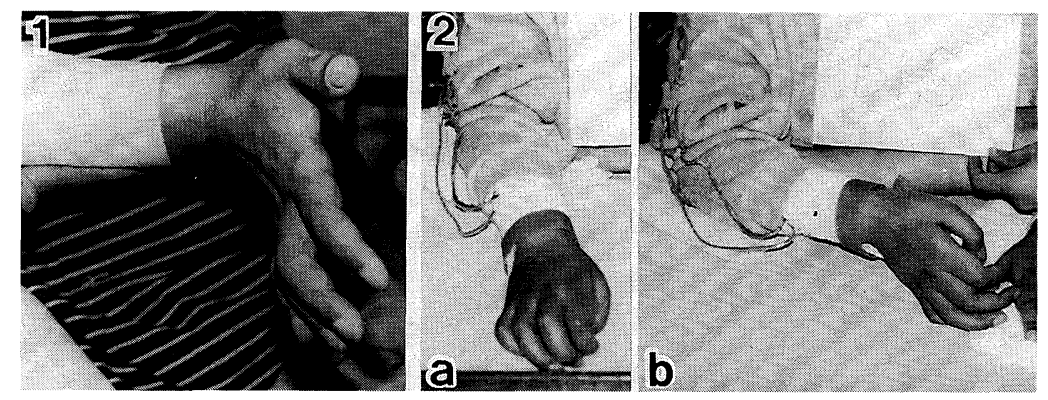

Fig. 1. Movements of a paralyzed upper extremity induced by FES to the supinator. Not only forearm supination but also wrist, finger and thumb extension, and thumb abduction were induced by an activation of the posterior interosseus nerve.

Fig. 2. Movements induced by FES to the biceps brachii (short head) in a quadriplegic patient. Before FES, the upper extremity was put on a table of a wheel chair with the elbow slightly flexed and the forearm pronated (a). FES to the biceps brachii resulted in simultaneous inductions of elbow flexion and forearm supination (b).

forearm supinator (Basmajian 1982; Clemente 1985; Williams et al. 1989). We have confirmed that electrical activation of this muscle induced both elbow flexion and forearm supination in normal volunteers (Naito et al. 1994) and quadriplegic patients (Fig. 2). On the other hand, electromyographic (EMG) studies indicated that this muscle did not act during elbow movements including extension and flexion when the forearm was pronated (Sullivan et al. 1950; Basmajian and Latif 1957; Pauly et al. 1967; Basmajian and Deluca 1985; Naito et al. 1991, 1994). In this study, we controlled flexion and extension with the forearm prone using FES to the brachialis, brachioradialis and triceps brachii. Then supination was controlled using additional FES to the biceps brachii.

\section{Subject And Methods}

FES to the biceps brachii, the elbow extensors (lateral or medial heads of the triceps brachii) and flexors (the brachialis or brachioradialis) was performed in the right upper extremity of a patient (male, 26 years of age) with incomplete $\mathrm{C} 4$ quadriplegia. All modes of sensation and voluntary motor functions of the right upper extremity including the shoulder were lost by an accident of a fall two years and three months before. No apparent atrophy was observed in the right hand, and the tendon reflexes of the biceps and triceps brachii muscles were pathologically accentuated. Spasticity was observed in the elbow flexors and extensors and also the forearm pronators and supinators. The spasticity usually predominated in the extensors and the pronators. By passive movements, the range of flexion and extension of the elbow was almost full (125 degrees: from 0 to 125 degrees of flexion) while that of pronation and supination of the forearm 
was small and limited (100 degrees: from 70 degrees of pronation to 30 degrees of supination) probably due to shortening of the muscles. The patient complained of dysesthesia and/or paresthesia in the hand. The intramuscular electrodes had been already implanted in the upper extremity for activating the muscles below the shoulder using our FES system (Naito et al. 1990). For the FES, we used amplitude modulating rectangular pulses $(-20-0 \mathrm{~V})$ with a duration of $0.2 \mathrm{msec}$, of frequency was $20 \mathrm{~Hz}$ (Handa and Hoshimiya 1987).

Before the FES, the forearm was pronated (70 degrees of pronation) and the elbow was slightly flexed (20 degrees of flexion). During the FES, the upper arm was supported by a experimenter's hand. While keeping the elbow in a fixed angle using FES to the flexors and extensors, movements induced by additional FES to the biceps brachii, performed by changing the stimulation voltage from 0 to $-20 \mathrm{~V}$ for several seconds, were examined.

\section{ObSERVATIONS}

FES to the extensors and flexors provided elbow extension and flexion, of which the range was almost full (125 degrees) as well as in the passive movements, with keeping the forearm prone. However, FES to these muscles did not provide a steady maintenance of the elbow except in the maximum extension and flexion and additional FES to the biceps brachii always resulted in simultaneous inductions of supination and flexion. Then we tried to control supination with keeping
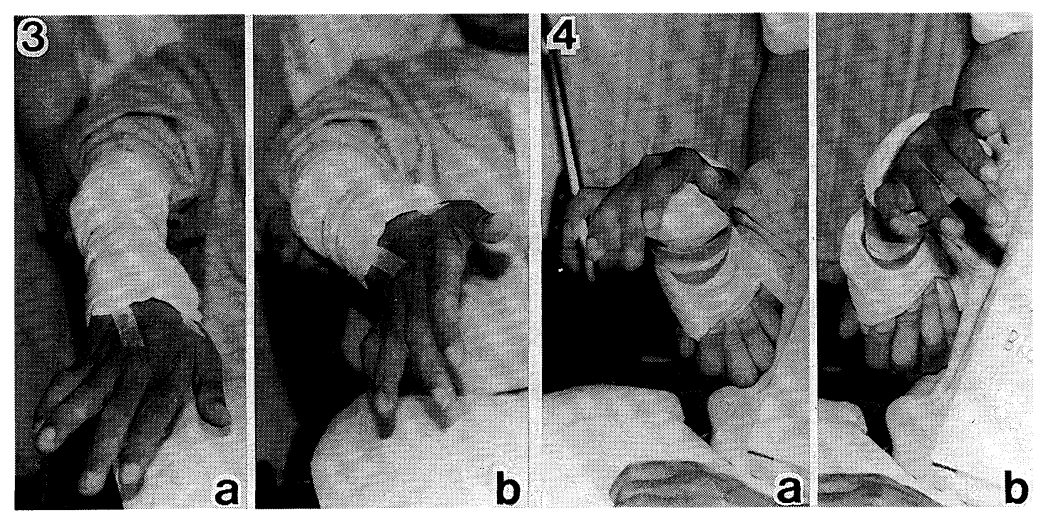

Fig. 3. Forearm supination induced by FES to the biceps brachii and the elbow extensors in a quadriplegic patient. The elbow was extended to the maximum extension by FES to the triceps brachii (lateral head) (a). Additional FES to the biceps brahii resulted in induction of supination with keeping the elbow extended (b).

Fig. 4. Forearm supination induced by FES to the biceps brachii and the other elbow flexors in a quadriplegic patient. The elbow was flexed to the maximum flexion by FES to the brachialis (a). Additional FES to the biceps brachii induced supination with keeping the elbow flexed (b). 
the elbow in the maximum extension and flexion. FES to the extensors extended the elbow to the maximum extension with keeping the forearm prone (Fig. 3-a). While keeping the elbow extended by FES to the extensors, additional FES to the biceps brachii resulted in supination of the forearm without an induction of flexion (Fig. 3-b). The maximum flexion of the elbow was also induced by FES to the flexors with keeping the forearm prone (Fig. 4-a). While keeping the elbow flexed by FES to the flexors, supination was induced by additional FES to the biceps brachii in maintenance of flexion (Fig. 4-b). The ranges of supination induced by FES to the biceps brachii in elbow extension and flexion were approximately 50 and 30 degrees from the forearm prone position, respectively.

\section{Discussion}

In the present study, FES to the elbow flexors and extensors did not provide a steady maintenance of the elbow except in the maximum extension and flexion. From EMG study of elbow flexion and extension, we have found that the flexors were usually active during both the flexion and extension phases but the triceps brachii, considered as the chief extensor, was almost inactive during the movement except at the period of the maximum extension (Naito et al. 1991). As regards the triceps brachii, Basmajian (1982) described that it is a powerful extensor but, on most occasions, it enjoys the assistance of gravity. Thus it seems that a delicate balance of forces of the muscles and gravity provides a maintenance of the elbow joint. Since additional FES to the biceps brachii disturbs the balance, more ingenious FES to the muscles, i.e., turning down FES to the flexors and/or turning up FES to the extensors accompanied with additional FES to the biceps brachii, was required to control supination without an induction of flexion.

The present study demonstrated that FES to the biceps brachii, and the extensors and flexors provided supination in maintenance of the maximum elbow extension and flexion, respectively. However, the ranges of the movements were smaller than that of the passive supination, especially in a maintenance of the flexion. Because the biceps brachii is slackened by elbow flexion, FES to this muscle must become less effective with increasing a degree of flexion. Furthermore, a reduction of the muscle strength caused by a disused atrophy usually occurs in these patients. These factors may provide a limitation of supination by FES to the biceps brachii. Although a range of the movements was still small and limited, the present study indicates a possibility of FES to the biceps brachii for controlling forearm supination in paralyzed upper extremities.

\section{Acknowledgments}

This work was partly supported by Grant-in Aid for Scientific Research from the Ministry of Education, Science and Culture of Japan (No. 61890007, 62460130, 63850081, 01870001). We wish to thank Mr. Michio Miyata and Miss Kumiko Yokouchi for their excellent assistance. Thanks are also tendered to members of our FES research group. 


\section{References}

1) Basmajian, J.V. (1982) Primary Anatomy. 8th ed., Williams and Wilkins, Baltimore, pp. 149-155.

2) Basmajian, J.V. \& Deluca, C.J. (1985) Muscle Alive: Their Functions Revealed by Electromyography. 5th ed., Williams and Wilkins, Baltimore, pp. 265-289.

3) Basmajian, J.V. \& Latif, A. (1957) Integrated actions and functions of the chief flexors of the elbow. J. Bone Joint Surg., 39-A, 1106-1118.

4) Clemente, C.D. (1985) Gray's Anatomy of the Human Body. 30th American ed., Lea and Febiger, Philadelphia, pp. 527-528.

5) Handa, Y. \& Hoshimiya, N. (1987) Functional electrical stimulation for the control of the upper extremities. Med. Prog. Technol., 12, 51-63.

6) Handa, Y., Hoshimiya, N., Iguchi, Y. \& Oda, T. (1989) Developments of percutaneous intramuscular electrode for multi-channel FES system. IEEE Trans. Biomed. Eng., 36, 705-710.

7) Hoshimiya, N., Naito, A., Yajima, M. \& Handa,Y. (1989) A respiration controlled FES system for muti-joint upper extremity. IEEE Trans. Biomed. Eng., 36, 754-760.

8) Naito, A., Yajima, M., Fukamachi, H., Ushikoshi, K., Ichie, M., Handa, Y. \& Hoshimiya, N. (1990) Creation of neuromuscular stimulation data for functional electrical stimulation: Control of paralyzed elbow movements. Jpn. J. Med. Elect. Biol. Eng., 28, 50-55. (in Japanese with English abstract)

9) Naito, A., Shimizu, Y., Handa, Y., Ichie, M. \& Hoshimiya, N. (1991) Functional anatomical sutdies of the elbow movements. I. Electromyographic (EMG) analysis. Okajimas Folia Anat. Jpn., 68, 283-288.

10) Naito, A., Yajima, M., Fukamachi, H., Ushikoshi, K., Handa, Y., Hoshimiya, N. \& Shimizu, Y. (1994) Electrophysiological studies of the biceps brachii activities in supination and flexion of the elbow joint. Tohoku J. Exp. Med., 173, 261-269.

11) Pauly, J.E., Rushing, J.L. \& Scheving, L.E. (1967) An electromyographic study of some muscles crossing the elbow joint. Anat. Rec., 159, 47-54.

12) Sullivan, W.E., Mortensen, O.A., Miles, M. \& Green, L.S. (1950) Electromyographic studies of m. biceps brachii during normal voluntary movement at the elbow. Anat. Rec., 107, 243-252.

13) Williams, P.L., Warwick, R., Dyson, M. \& Bannister, L.H. (1989) Gray's Anatomy, 37th ed., Churchill Livingstone, Edinburgh, pp. 614-615. 